\title{
Viewpoint
}

\section{Aiming with bubbles}

\author{
Claus-Dieter Ohl \\ School of Physical and Mathematical Sciences, Division of Physics and Applied Physics, Nanyang Technological University, \\ 21 Nanyang Link, 637371 Singapore
}

Published August 9, 2010

\begin{abstract}
An alternative to a needle for penetrating cells may be found in the precise and forceful fluid jet created by two bubbles expanding and collapsing in tandem.
\end{abstract}

Subject Areas: Optics, Fluid Dynamics, Biological Physics

\author{
A Viewpoint on: \\ Pulsating Tandem Microbubble for Localized and Directional Single-Cell Membrane Poration \\ G. N. Sankin, F. Yuan, and P. Zhong \\ Physical Review Letters 105, 0781012010 - Published August 9, 2010
}

The bubbles that form in a cavitating liquid are mostly empty voids that can accelerate the surrounding fluid to very high speeds, in some cases focusing the liquid flow into needle thin jets. The military, for example, harnesses this effect to penetrate and sink large vessels with the huge cavitation bubble created with an underwater explosion. Now, a miniaturized (and more peaceful) version of a cavitation driven jet is offering exciting new possibilities in biology. Georgii Sankin, Fang Yuan, and Pei Zhong at Duke University in the US have designed a tandem-bubble technique to poke through the membrane of a single biological cell with a micrometer sized jet. Their in-depth study, which is reported in Physical Review Letters, demonstrates that with the proper control of cavitation, it is possible to engineer complex, localized, and repeatable flows on the micrometer scale [1].

Poking through an elastic membrane is most effective when done fast, with skilled force and a pointed needle tip. These rules hold for both the doctor performing an injection into a patient's skin and the scientist delivering a drug through the protective membrane of a single cell. If the force is too weak, the needle will merely deform the cell's membrane without penetrating, while if the needle is too wide, the hole in the cell membrane may never heal. Thus certain skills and the right tools are necessary to poke and inject cells without killing them in the process.

Over the course of several years, the Duke researchers have developed a method to penetrate membranes using the tools of optics, instead of needles. The antagonists of their experiments are two pulsed Neodymium:YAG lasers, one operating at a wavelength of $1064 \mathrm{~nm}$ and the other at $532 \mathrm{~nm}$. The lasers are tightly focused and absorbed by Trypan blue dye molecules in a bath of water. Liquid at the focus of the high-intensity laser light vaporizes and a vapor bubble expands explosively, reaches maximum size, and then collapses back into the liquid

DOI: $10.1103 /$ Physics.3.65

URL: http://link.aps.org/doi/10.1103/Physics.3.65 due to the atmospheric pressure.

A single bubble from a single laser creates a purely radial flow, with an outward flow during bubble expansion and an inward flow during collapse. Yet if a second bubble is created exactly at the time when the first bubble starts to shrink, the radial symmetry is broken and a new flow pattern forms: the outward pressure created by the second (expanding) bubble combines with the inward pressure from the first (collapsing) bubble (see Fig. 1). As the first bubble collapses, a V-shaped singularity forms on its side, which produces a thin, high-speed fluid jet. Similar jets emerge in oscillating liquid-gas interfaces [2, stones plummeting into a liquid [3, and shrinking and translating bubbles like those the Duke team have produced [4, 5].

Creating this jetting flow was only the starting point of the Duke team's research; the more difficult part was observing and manipulating it. To create flows on a length scale appropriate for poking the membrane of a cell, Sankin et al. generated bubbles that expanded to a maximum radius of $R_{M A X} \approx 25 \mu \mathrm{m}$. The lifetime of these vapor-filled bubbles can be estimated roughly from the classical Rayleigh collapse time $T_{C} \approx R_{M A X}\left(\rho / P_{0}\right)^{1 / 2}$, where $P_{0}$ is the atmospheric pressure, and $\rho$ the liquid density [6]. Inserting numbers, one sees that bubbles of this size collapse within microseconds and observing them requires a high-speed camera. They recorded the bubble dynamics by taking a limited number of frames with very short shutter times (1 million frames/s), while 100 frames of the flow field were captured with a CCD high-speed camera. The flow field obtained from the displacement of the particles reveals jet velocities of $10 \mathrm{~m} / \mathrm{s}$ (22.5 miles/hour). The true maximum velocity is likely higher, as the thinnest and fastest parts cannot be resolved. Interestingly, the high-speed jet drags the surrounding fluid, which leads to a pronounced circulating flow that is clockwise on one side of the jet and counter- 

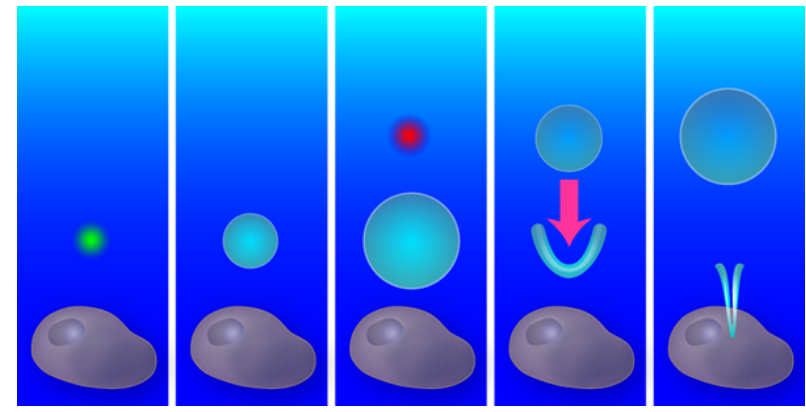

FIG. 1: The timed expansion and collapse of two bubbles creates a liquid jet that can penetrate a fine hole in the membrane of a cell. From left to right: A laser (green circle) focused inside a water bath locally vaporizes the liquid, creating an expanding bubble (light blue). Just after the first bubble reaches its maximum size, a second laser (red circle) generates another bubble. As the second bubble expands and the first bubble collapses, a rush of liquid forms along the vertical line (pink arrow) between the two, creating a high-speed liquid jet that accelerates toward the cell with enough force to penetrate the membrane. (Illustration: Alan Stonebraker)

clockwise on the other side. The Duke group measured rotations rates of $50,000 \mathrm{~s}^{-1}$ in the two counter-rotating vortices; a truly microscopic blender!

Having visualized the flow, the Duke team tackled single-cell penetration experiments with a determination to obtain statistically significant data. They chose an adherent carcinoma mammary cell line as their target and used the Trypan blue dye molecules dissolved in the extracellular liquid to mark the cell. (The membrane is impermeable to the dye molecules unless a pore is created.) Once inside the cell, the dye stains the cytoplasm and nucleus a darkish blue. The group recorded the uptake of this dye after the tandem bubble action with another high-speed camera (Video 1 ).

To find the optimal flow field for liquid injection, the team probed a range of bubble configurations and flow patterns: the radial (non-jet) flow from a single bubble, the tandem bubble with the first bubble jetting towards the cell or the second bubble (as shown in Fig. 1), and the tandem bubble aligned such that the jet doesn't project toward the cell. They also varied the distance between the bubble configuration and the cell, from $10 \mu \mathrm{m}$ to $40 \mu \mathrm{m}$. After testing each of these configurations with 15-25 cells, the winner emerged: the cell had a statistically significant increase in Trypan blue uptake ( $p$-value $<0.05)$ for the tandem bubble configuration that was initially $20 \mu \mathrm{m}$ away from the cell with the first bubble jetting towards the cell.

The tandem bubbles form an effective microscopic "needle"; but could the high-speed flow actually damage the cell? Unfortunately, Trypan blue is toxic, so every cell in this study that absorbed the dye eventually died. Sankin et al. obtained some insight into how the cell membrane was affected by taking a picture of a punctured cell at the site of penetration: Within $10 \mathrm{~s}$ after

DOI: 10.1103/Physics.3.65

URL: http://link.aps.org/doi/10.1103/Physics.3.65

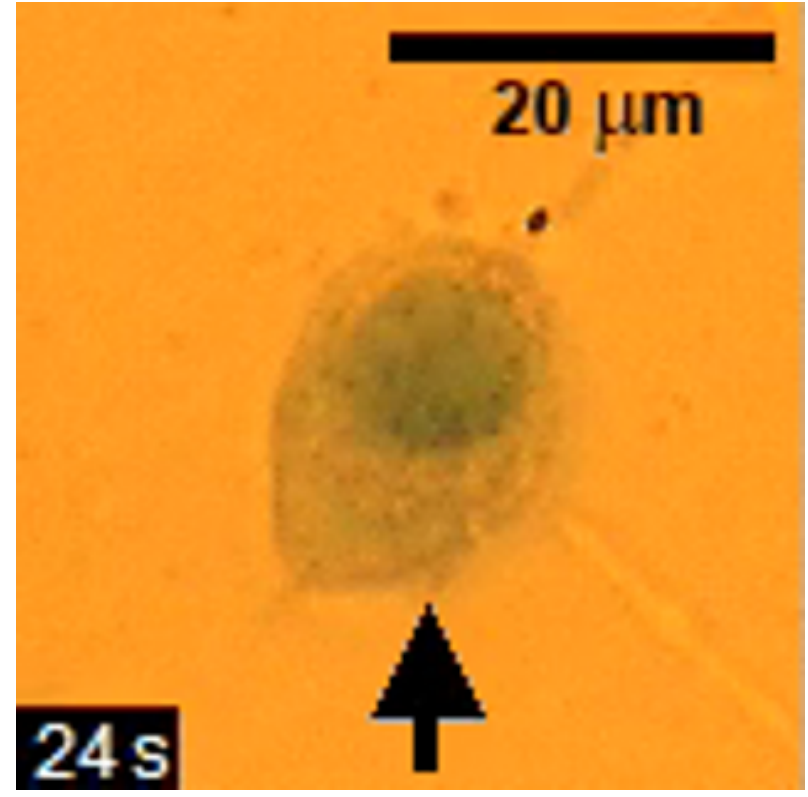

VIDEO 1: Penetration of Tryptan blue into a carcinoma mammary cell after it is punctured with a liquid jet. (Video: Sankin et al.[1]) Video available at: http://physics.aps. org/articles/v/\#video.

the jet plunged through the membrane, they treated the cell chemically to stop all cellular repair mechanisms and marked the position of the bubble. They later scanned the cell membrane for holes using an electron microscope and showed that the pores at the site where the jet impacted were about $200 \mathrm{~nm}$ in diameter. (For comparison, mammary cells are about 10-20 $\mu \mathrm{m}$ wide.)

Although this study is not the first demonstrating that jetting bubbles can rupture cell membranes it is, to my knowledge, the most complete experiment that characterizes the flow, measures the uptake on single cells, and obtains a one-to-one correlation between the observed jet and the measured membrane pore. The Duke team's method qualifies as a new technique for drug delivery to single cells, and if engineered further may become a tool for biologists for particularly delicate cell lines and perhaps stem cells. From a physics perspective I see a more important implication of this work: There is exciting potential to use laser generated cavitation bubbles to precisely control fluid flow and very fine jets on submicrometer length scales.

\section{References}

[1] G. N. Sankin, F. Yuan, and P. Zhong, Phys. Rev. Lett. 105, 078101 (2010).

[2] B. W. Zeff, B. Kleber, J. Fineberg, and D. P. Lathrop, Nature 403, 401 (2000).

[3] S. Gekle, M. J. Gordillo, D. van der Meer, and D. Lohse, Phys. Rev. Lett. 102, 034502 (2009).

[4] W. Lauterborn and H. Bolle, J. Fluid Mech. 72, 391 (1975). 
[5] J. R. Blake, M. C. Hooton, P. B. Robinson, and R. P. Tong, Philos. Trans. R. Soc. London 355, 537 (1997).

[6] C. E. Brennen, Cavitation and Bubble Dynamics, (Oxford
University Press, New York, 1990); ebook available at http:

//resolver.caltech.edu/CaltechBO0K:1995.001.

\section{About the Author}

\section{Claus-Dieter Ohl}

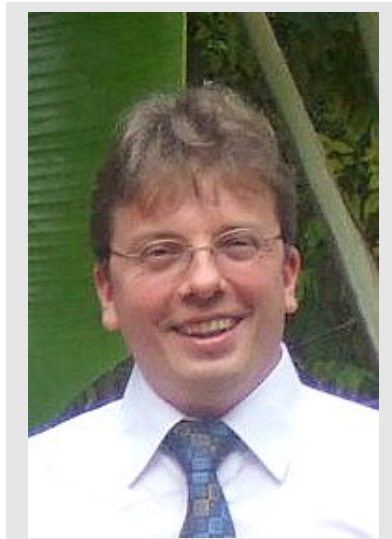

Claus-Dieter Ohl is Assistant Professor at the Nanyang Technological University and Adjunct Professor at the Institute of High Performance Computing in Singapore. Previously, he worked as a postdoctoral fellow at Johns Hopkins University, US, and at the University of Twente in the Netherlands. His research is focused on experimental fluid mechanics, in particular utilizing lasers, shock waves, and cavitation bubbles for applications in medical therapy, biology, and engineering. He is currently interested in sonoluminescence and sonochemistry in microfluidics. Besides that, he is curious to figure out how ultrasonic cleaning really works. 